\title{
VOLUME 49, 2005
}

\section{CONTRIBUTOR INDEX}

\section{A}

Abbes I (see Mrad et al). 2005;49:427-430

Adabă̆ A (see Köybaşıŏlu et al). 2005;49:173-176

Adachi J (see Inagawa et al). 2005;49:452-456

Adachi M (see Nakashima et al). 2005;49:87-91

Adesina AO (see Jogai et al). 2005;49:483-488

Afify A, Zhou H, Howell L, Paulino AFG. Diagnostic Utility of GLUT-1 Expression in the Cytologic Evaluation of Serous Fluids. 2005;49:621-626

Afroz N, Khan N, Chana RS. Yolk Sac Tumor of Childhood: Diagnosis on Fine Needle Aspiration Biopsy. 2005;49:697-698 (Letter)

Agarwal A (see Bhadani et al). 2005;49:228-230 (Letter) Agarwal A (see Bhadani et al). 2005;49:347-348 (Letter) Agarwal M (see Bhadani et al). 2005;49:114-116 (Letter) Agarwala S (see Iyer et al). 2005;49:355-364

Ahmed MS (see Das et al). 2005;49:65-70

Ajit D (see Dighe and Ajit). 2005;49:416-420

Akinremi TO, Nazeer S, Tötsch M. Reduced Alcohol Use in the Staining of Pap Smears: A Satisfactory, Low-Cost Protocol for Cervical Cancer Screening. 2005;49:169-172

Al-Jassar A (see Jogai et al). 2005;49:483-488

Alonso de la Campa J (see Pinto Blázquez et al). 2005; 49:653-655

Amanguno HG (see Jogai et al). 2005;49:483-488

Amaral RG, Zeferino LC, Hardy E, Westin MCdA, Martinez EZ, Montemor EB. Quality Assurance in Cervical Smears: 100\% Rapid Rescreening vs. 10\% Random Rescreening. 2005;49:244-248

Andorno S (see Boldorini et al). 2005;49:235-243

Andreadis C (see Malle et al). 2005;49:11-16

Andres L, Etxegarai L, Ibarrola R, Hermosa C, López JI. Fasciitislike Papillary Carcinoma of the Thyroid Gland. 2005;49:462-463 (Letter)

Anshu, Shivkumar VB, Gangane N, Sharma S. Metaplastic Carcinoma of the Breast in an HIV-Positive Patient: A Case Report. 2005;49:204-206

Antúnez JR (see Suárez-Peñaranda et al). 2005;49: $530-532$

Anuradha, Sinha A, Ramrakhiani D. Primary NonHodgkin's Lymphoma of the Breast: A Case Report. 2005;49:661-665

Aron M (see Mathur et al). 2005;49:567-570

Arora VK (see Malhotra et al). 2005;49:580-582 (Letter)

Astudillo Gonzalez A (see Pinto Blázquez et al). 2005; 49:653-655
Athanassiadou P, Grapsa D. Fine Needle Aspiration of Borderline Ovarian Lesions: Is It Useful? 2005; 49:278-285

Atkinson EN (see Cantor et al). 2005;49:405-415

Au VSC (see Chuah et al). 2005;49:75-80

Ayala AG (see Sun et al). 2005;49:525-529

Ayed FB (see Mrad et al). 2005;49:427-430

\section{B}

Baehner FL, Ng B, Sudilovsky D. Metastatic Balloon Cell Melanoma: A Case Report. 2005;49:543-548

Bagheri H (see Kumar et al). 2005;49:445-448

Bakaris S, Ozdemir M, Is1k IO, Buyukbese MA, Ozdemir G. Impression Cytology Changes and Corneoconjunctival Calcification in Patients with Chronic Renal Failure. 2005;49:1-6

Bakotic WL, Willis D, Birdsong G, Tadros TS. Anal Cytology in an HIV-Positive Population: A Retrospective Analysis. 2005;49:163-168

Baliga M (see Guo et al). 2005;49:132-138

Banek L (see Banek et al). 2005;49:627-633

Banek T, Banek L, Pezerović-Panijan R. Morphology of Healthy Human Parathyroid Glands in Cytologic Smears. 2005;49:627-633

Barco D (see Boldorini et al). 2005;49:235-243

Barker BJ (see Zardawi et al). 2005;49:224-226 (Letter)

Barnhart A (see Taylor et al). 2005;49:673-676

Basu D (see Garbyal et al). 2005;49:58-60

Batoroev YK, Nguyen G-K. Rhabdoid Meningioma Diagnosed by Imprint Cytology. 2005;49:464-465 (Letter)

Bavi P, Shet T, Gujral S. Malignant Melanoma of Mediastinum Misdiagnosed as a Spindle Cell Thymoma in a Fine Needle Aspirate: A Case Report. 2005;49:424-426

Bedayat GR (see Daneshbod et al). 2005;49:7-10

Beksaç MS (see Demirezen et al). 2005;49:257-261

Ben Romdhane K (see Mrad et al). 2005;49:427-430

Benedet JL (see Cantor et al). 2005;49:405-415

Bewtra C, Xie Q, Soundararajan S, Gatalica Z, Hatcher L. Genital Human Papillomavirus Testing by in Situ Hybridization in Liquid Atypical Cytologic Materials and Follow-up Biopsies. 2005;49: 127-131

Bhadani PP, Agarwal A, Sah SP, Sharma U. Fine Needle Aspiration Diagnosis of Ectopic Thyroid 
with Laboratory Features of Hyperthyroidism. 2005;49:228-230 (Letter)

Bhadani PP, Sah SP, Agarwal M, Tewari A. Fine Needle Aspiration Diagnosis of Congenital Langerhans Cell Histiocytosis of the Lymph Nodes with Isolated Skin Involvement. 2005;49:114-116 (Letter)

Bhadani PP, Singh RK, Sah SP, Agarwal A. Sinus Histiocytosis with Massive Lymphadenopathy: Diagnosis by Fine Needle Aspiration. 2005;49: 347-348 (Letter)

Bhalla P (see Sodhani et al). 2005;49:634-638

Bhatia A (see Malhotra et al). 2005;49:580-582 (Letter)

Bhuta S (see Wiriosuparto et al). 2005;49:309-313

Bibbo M (see Wood et al). 2005;49:291-296

Birdsong G (see Bakotic et al). 2005;49:163-168

Blasi AD (see Ferrara et al). 2005;49:467-468 (Letter)

Bleggi-Torres LF (see Collaço et al). 2005;49:694-696 (Letter)

Bobhate SK (see Jungare et al). 2005;49:600-604

Bohrer PL, Filho MS, Paiva RL, da Silva IL, Rados PV. Assessment of Micronucleus Frequency in Normal Oral Mucosa of Patients Exposed to Carcinogens. 2005;49:265-272

Bolat F (see Uguz et al). 2005;49:144-148

Boldorini R (see Bozzola et al). 2005;49:213-218

Boldorini R, Brustia M, Veggiani C, Barco D, Andorno S, Monga G. Periodic Assessment of Urine and Serum by Cytology and Molecular Biology as a Diagnostic Tool for BK Virus Nephropathy in Renal Transplant Patients. 2005;49:235-243

Bolick D (see Qureshi et al). 2005;49:120-126

Boon ME (see Rao et al). 2005;49:249-256

Boon ME, Ouwerkerk-Noordam E, Suurmeijer AJH, Kok LP. Diagnostic Parameters in Liquid-Based Cervical Cytology Using a Coagulant Suspension Fixative. 2005;49:513-519

Boon ME. Dr. Darier's Lesson: It Can Be Advantageous to the Patient to Ignore Evident Cytonuclear Changes. 2005;49:469-470

Bozzetti C (see Nizzoli et al). 2005;49:27-30

Bozzola C, Boldorini R, Ramponi A, Monga G. Fine Needle Aspiration Cytology in the Diagnosis of Non-Hodgkin's Lymphomas of the Muscle: A Report of 2 Cases. 2005;49:213-218

Branca M (see Longatto-Filho et al). 2005;49:500-506

Branca M, Morosini P, Severi P, Eržen M, Di Benedetto C, Syrjänen K. New Statistical Software for Intralaboratory and Interlaboratory Quality Control in Clinical Cytology: Validation in a Simulation Study on Clinical Samples. 2005;49:398404

Brown HM, Wilkinson EJ. Cytology of Secondary Vulvar Paget's Disease of Urothelial Origin: A
Case Report. 2005;49:71-74

Brustia M (see Boldorini et al). 2005;49:235-243

Burstein D (see Jaffer et al). 2005;49:323-326

Burstein DE (see Szporn et al). 2005;49:262-264

Burstein DE (see Szporn et al). 2005;49:387-390

Burstein DE (see Wu and Burstein). 2005;49:692-694 (Letter)

Buyukbese MA (see Bakaris et al). 2005;49:1-6

\section{C}

Caleo A (see Fulciniti et al). 2005;49:495-499

Cantor SB, Atkinson EN, Cardenas-Turanzas M, Benedet JL, Follen M, MacAulay C. Natural History of Cervical Intraepithelial Neoplasia: A Metaanalysis. 2005;49:405-415

Capron M (see Duboucher et al). 2005;49:273-277

Caraway NP (see Liu et al). 2005;49:157-162

Caraway NP (see Sun et al). 2005;49:525-529

Cardenas-Turanzas M (see Cantor et al). 2005;49: 405-415

Chana RS (see Afroz et al). 2005;49:697-698 (Letter)

Chang T-C (see Shih et al). 2005;49:383-386

Chang T-C (see Shih et al). 2005;49:584-585 (Letter)

Chang T-C, Wu S-L, Hsiao Y-L. Medullary Thyroid Carcinoma: Pitfalls in Diagnosis by Fine Needle Aspiration Cytology and Relationship of Cytomorphology to RET Proto-oncogene Mutations. 2005; 49:477-482

Chatzistamou I, Kondi-Pafiti A, Politi E, Koutselini H, Schally AV. Detection of Growth HormoneReleasing Hormone in Cytologic Specimens of Primary Breast Carcinoma. 2005;49:108-109 (Letter)

Chen KTK. Extraneous Cells of Hepatic Origin in Adrenal Fine Needle Aspiration as a Diagnostic Pitfall: A Case Report. 2005;49:449-451

Chen S-F (see Nieh et al). 2005;49:31-37

Chen X (see Szporn et al). 2005;49:262-264

Chen X (see Szporn et al). 2005;49:387-390

Chon S-H (see Oh et al). 2005;49:343-344 (Letter)

Chu YC (see Kim et al). 2005;49:644-649

Chuah KL, Hwang JSG, Ng SB, Tan PH, Poh WT, Au VSC. Cytologic Features of Cribriform-Morular Variant of Papillary Carcinoma of the Thyroid: A Case Report. 2005;49:75-80

Cibas ES (see O'Connell and Cibas). 2005;49:187-190

Clark AH (see Khirwadkar and Clark). 2005;49:344345 (Letter)

Clary KM, Condel JL, Liu Y, Johnson DR, Grzybicki DM, Raab SS. Interobserver Variability in the Fine Needle Aspiration Biopsy Diagnosis of Follicular Lesions of the Thyroid Gland. 2005;49:378382 
Collaço LM, de Noronha L, Bleggi-Torres LF, Pinheiro DL. Quality Control in Cervical Cancer Screening: Brazilian Experience. 2005;49:694-696 (Letter)

Collins BT (see Elmberger et al). 2005;49:223-224 (Letter)

Collins BT (see Ezenekwe et al). 2005;49:549-553

Condel JL (see Clary et al). 2005;49:378-382

Costabel U (see Uzaslan et al). 2005;49:154-156 [erratum 2005;49(5):127A]

Crespo M (see Velasco et al). 2005;49:585-586 (Letter)

\section{D}

da Silva IL (see Bohrer et al). 2005;49:265-272

Dalena AM (see Ferrara et al). 2005;49:467-468 (Letter)

Dall' Agnol M (see Mattosinho de Castro Ferraz et al). 2005;49:639-643

Daneshbod Y (see Kumar et al). 2005;49:445-448

Daneshbod Y, Monabati A, Kumar PV, Rastegar M. Paratesticular Spindle Cell Rhabdomyosarcoma Diagnosed by Fine Needle Aspiration Cytology: A Case Report. 2005;49:331-334

Daneshbod Y, Monabati A, Kumar PV, Taghipoor M, Bedayat GR. Intraoperative Cytologic Crush Preparation Findings in Craniopharyngioma: A Study of 12 Cases. 2005;49:7-10

Daneshbood Y (see Geramizadeh et al). 2005;49:110_ 111 (Letter)

Das DK, Haji BE, Ahmed MS, Hossain MNR. Myoepithelioma of the Parotid Gland Initially Diagnosed by Fine Needle Aspiration Cytology and Immunocytochemistry: A Case Report. 2005; 49:65-70

Das DK. Nucleolar Positivity for CD20: A Diagnostic Aid in Neoplasms of T-Cell Lineage? 2005;49: 365-372

Dávila RM (see Faulkner-Jones et al). 2005;49:297308

de la Campa JA (see Velasco et al). 2005;49:585-586 (Letter)

de Lang A, Wilander E. Sensitivity of HPV Tests on Stained vs. Unstained Cervical Smears. 2005;49: 595-599

de Noronha L (see Collaço et al). 2005;49:694-696 (Letter)

Dei-Cas E (see Duboucher et al). 2005;49:273-277

Delgado-Viscogliosi P (see Duboucher et al). 2005;49: 273-277

Demirezen Ş, Kaya D, Beksaç MS. Cytologic Findings in Pap Smears with Actinomyces-like Organisms. 2005;49:257-261
Derchain SFM (see Longatto-Filho et al). 2005;49: 500-506

Deshpande A, Munshi M. Cytology of Papillary Solid-Cystic Neoplasm of the Pancreas Presenting as an Extrapancreatic Mass: A Case Report. 2005; 49:81-86

Destouni C (see Malle et al). 2005;49:11-16

Destouni H (see Valeri et al). 2005;49:61-64

Dey P (see Jogai et al). 2005;49:483-488

Dharan M. Metastatic Minor Salivary Gland Colloid Carcinoma in the Parotid Region After Parotidectomy for Pleomorphic Adenoma: A Case Report. 2005;49:101-104

Di Benedetto C (see Branca et al). 2005;49:398-404

di Loreto C (see Mattosinho de Castro Ferraz et al). 2005;49:639-643

Dighe S, Ajit D. Collection Devices for Cervicovaginal Cytology: A Comparison. 2005;49:416-420

Doğan N (see Üçok et al). 2005;49:38-42

Domínguez AN (see Suárez-Peñaranda et al). 2005; 49:530-532

Driss M (see Mrad et al). 2005;49:427-430

Duboucher C, Gerbod D, Noël C, Durand-Joly I, Delgado-Viscogliosi P, Leclerc C, Pham S, Capron M, Dei-Cas E, Viscogliosi E. Frequency of Trichomonads as Coinfecting Agents in Pneumocystis Pneumonia. 2005;49:273-277

Durand-Joly I (see Duboucher et al). 2005;49:273-277

Dvorak K (see Kren et al). 2005;49:441-444

\section{$\mathbf{E}$}

Ebsen M (see Uzaslan et al). 2005;49:154-156 [erratum 2005;49(5):127A]

Edwards PC, Wasserman P. Evaluation of Cystic Salivary Gland Lesions by Fine Needle Aspiration: An Analysis of 21 Cases. 2005;49:489-494

Elmberger PG, Sköld CM, Collins BT. Fine Needle Aspiration Biopsy of Intrabronchial Granular Cell Tumor. 2005;49:223-224 (Letter)

Elsayed EM (see Jayaram and Elsayed). 2005;49:520524

Elsayed EM (see Jayaram and Elsayed). 2005;49:605610

Emanuel P (see Jaffer et al). 2005;49:323-326

Ersoz C (see Uguz et al). 2005;49:144-148

Eržen M (see Branca et al). 2005;49:398-404

Eržen M (see Longatto-Filho et al). 2005;49:500-506

Esteban J-G (see Martínez-Girón et al). 2005;49:578580 (Letter)

Etxegarai L (see Andres et al). 2005;49:462-463 (Letter)

Ezenekwe AM, Collins BT, Ponder TB. Mixed Osteoclastic/Pleomorphic Giant Cell Tumor of the 
Pancreas: A Case Report. 2005;49:549-553

\section{$\mathbf{F}$}

Fatheezadeh P (see Geramizadeh et al). 2005;49:666668

Faulkner-Jones BE, Foster WJ, Harbour JW, Smith ME, Dávila RM. Fine Needle Aspiration Biopsy with Adjunct Immunohistochemistry in Intraocular Tumor Management . 2005;49:297-308

Feres CL (see Mattosinho de Castro Ferraz et al). 2005;49:639-643

Fernández-Palacín A (see Robles-Frías et al). 2005;49: $149-153$

Ferns S (see Kumar et al). 2005;49:111-112 (Letter)

Ferrara G, Dalena AM, Goglia P, Blasi AD. Glycogen-Rich Clear Cell Carcinoma of the Breast: A Chameleon? 2005;49:467-468 (Letter)

Filho MS (see Bohrer et al). 2005;49:265-272

Follen M (see Cantor et al). 2005;49:405-415

Foody W (see Hoda et al). 2005;49:199-203

Fortunato A (see Fulciniti et al). 2005;49:495-499

Foster WJ (see Faulkner-Jones et al). 2005;49:297-308

Franciosi V (see Nizzoli et al). 2005;49:27-30

French CA (see Oliveira and French). 2005;49:587594

Freudenberg N (see Uzaslan et al). 2005;49:154-156 [erratum 2005;49(5):127A]

Fu E (see Nieh et al). 2005;49:31-37

Fujiwara M, Horiguchi M, Inage Y, Horiguchi H, Satoh H, Kamma H. Combined Small Cell Carcinoma in the Peripheral Lung: Importance of Appropriate Sampling. 2005;49:575-578 (Letter)

Fukuda M (see Yamaguchi et al). 2005;49:207-212

Fulciniti F, Caleo A, Lepore M, Fortunato A, Vetrani A, Palombini L. Fine Needle Cytology of Endometriosis: Experience with 10 Cases. 2005;49:495499

\section{G}

Gadkari R (see Jungare et al). 2005;49:600-604

Gangane N (see Anshu et al). 2005;49:204-206

Garbyal RS, Basu D, Roy S, Kumar P. Cryptococcal Lymphadenitis: Report of a Case with Fine Needle Aspiration Cytology. 2005;49:58-60

Garg S (see Sodhani et al). 2005;49:634-638

Gatalica Z (see Bewtra et al). 2005;49:127-131

Geisinger KR (see Taylor et al). 2005;49:673-676

George PJ (see Madhavan et al). 2005;49:431-434

Geramizadeh B, Daneshbood Y, Karimi M. Cytology of Brain Metastasis of Yolk Sac Tumor. 2005;49: 110-111 (Letter)
Geramizadeh B, Kheirandish P, Fatheezadeh P, Jannesar R. Fine Needle Aspiration of Subcutaneous Masses Caused by Aspergillus: A Report of 2 Cases. 2005;49:666-668

Gerbod D (see Duboucher et al). 2005;49:273-277

Ghafari S (see Gu et al). 2005;49:471-476

Goel MM (see Jain et al). 2005;49:17-21

Goel MM (see Singh et al). 2005;49:345-347 (Letter)

Goglia P (see Ferrara et al). 2005;49:467-468 (Letter)

Gokdemir A (see Uguz et al). 2005;49:144-148

Goldfarb A (see Jaffer et al). 2005;49:323-326

Goncharuk V (see Kren et al). 2005;49:441-444

Gong Y, Jalali M, Staerkel G. Fine Needle Aspiration Cytology of a Thyroid Metastasis of Metaplastic Breast Carcinoma: A Case Report. 2005;49:327330

Gong Y, Ren R, Ordóñez NG, Sun X, Sneige N. Fine Needle Aspiration Cytology of Well-Differentiated Papillary Mesothelioma: A Case Report. 2005; 49:537-542

Gontijo R (see Longatto-Filho et al). 2005;49:500-506

González-Cámpora R (see Robles-Frías et al). 2005; 49:149-153

González-Peramato P (see Viguer et al). 2005;49:139_ 143

Grapsa D (see Athanassiadou and Grapsa). 2005;49: 278-285

Grzybicki DM (see Clary et al). 2005;49:378-382

Gu M, Ghafari S, Zhao M. Fluorescence in Situ Hybridization for HER-2/neu Amplification of Breast Carcinoma in Archival Fine Needle Aspiration Biopsy Specimens. 2005;49:471-476

Guazzi A (see Nizzoli et al). 2005;49:27-30

Gujral S (see Bavi et al). 2005;49:424-426

Günhan Ö (see Üçok et al). 2005;49:38-42

Guo M, Hu L, Martin L, Liu S, Baliga M, Hughson MD. Accuracy of Liquid-Based Pap Tests: Comparison of Concurrent Liquid-Based Tests and Cervical Biopsies on 782 Women with Previously Abnormal Pap Smears. 2005;49:132-138

Gupta S (see Sodhani et al). 2005;49:634-638

Gur R (see Sharma et al). 2005;49:335-338

Guzman J (see Uzaslan et al). 2005;49:154-156 [erratum 2005;49(5):127A]

\section{H}

Hadjileontis C (see Valeri et al). 2005;49:61-64

Haji BE (see Das et al). 2005;49:65-70

Hammes L (see Longatto-Filho et al). 2005;49:500506

Han JY (see Kim et al). 2005;49:644-649

Han Ü (see Köybaşığlu et al). 2005;49:173-176

Handa U, Khullar U, Mohan H. Pigmented Pheo- 
chromocytoma: Report of a Case with Diagnosis by Fine Needle Aspiration. 2005;49:421-423

Hansdak SG (see Sah et al). 2005;49:349-350 (Letter) Hara H, Oyama T, Suda K. New Criteria for Cytologic Diagnosis of Adenoid Cystic Carcinoma. 2005;49:43-50

Hara H, Suda K, Oyama T. Two Cytologic Patterns in Invasive Ductal Adenocarcinoma of the Pancreas. 2005;49:611-620

Harada T (see Takahashi et al). 2005;49:319-322

Harbour JW (see Faulkner-Jones et al). 2005;49:297308

Hardy E (see Amaral et al). 2005;49:244-248

Hashemi SB (see Shekarkhar et al). 2005;49:533-536

Hatcher L (see Bewtra et al). 2005;49:127-131

Hermanova M (see Kren et al). 2005;49:441-444

Hermosa C (see Andres et al). 2005;49:462-463 (Letter)

Hiatt RA (see Sawaya et al). 2005;49:391-397

Hirose T (see Inagawa et al). 2005;49:452-456

Hirschowitz S (see Wiriosuparto et al). 2005;49:309_ 313

Hoda RS, Minamiguchi S, Lewin DN, Foody W, Weselow G, Wildi SM. Granular Cell Tumor of the Biliary System: A Report of 2 Cases with Cytologic Diagnosis on Endoscopic Brushing. 2005;49: 199-203

Horiguchi H (see Fujiwara et al). 2005;49:575-578 (Letter)

Horiguchi M (see Fujiwara et al). 2005;49:575-578 (Letter)

Hossain MNR (see Das et al). 2005;49:65-70

Howell L (see Afify et al). 2005;49:621-626

Hsiao Y-L (see Chang et al). 2005;49:477-482

Hsiao Y-L (see Shih et al). 2005;49:383-386

Hsiao Y-L (see Shih et al). 2005;49:584-585 (Letter)

Hu L (see Guo et al). 2005;49:132-138

Huang Y (see Wood et al). 2005;49:291-296

Hughson MD (see Guo et al). 2005;49:132-138

Husseini SV (see Kumar et al). 2005;49:177-180

Hwang JSG (see Chuah et al). 2005;49:75-80

\section{I}

Ibarrola R (see Andres et al). 2005;49:462-463 (Letter)

Idris Z (see Madhavan et al). 2005;49:431-434

Ikeda K, Tate G, Suzuki T, Mitsuya T. Fine Needle

Aspiration Cytology of Primary Proximal-Type

Epithelioid Sarcoma of the Perineum: A Case

Report. 2005;49:314-318

Imamura Y (see Yamaguchi et al). 2005;49:207-212

Inagaki T (see Nakashima et al). 2005;49:87-91

Inagawa $\mathrm{H}$, Ishizawa $\mathrm{K}$, Mitsuhashi $\mathrm{T}$, Shimizu $\mathrm{M}$, Adachi J, Nishikawa R, Matsutani M, Hirose T.
Giant Invasive Pituitary Adenoma Extending into the Sphenoid Sinus and Nasopharynx: Report of a Case with Intraoperative Cytologic Diagnosis. 2005;49:452-456

Inage Y (see Fujiwara et al). 2005;49:575-578 (Letter) Ishizawa K (see Inagawa et al). 2005;49:452-456

Isık IO (see Bakaris et al). 2005;49:1-6

Iwana S (see Takahashi et al). 2005;49:319-322

Iyer VK (see Mathur et al). 2005;49:567-570

Iyer VK, Kapila K, Agarwala S, Verma K. Fine Needle Aspiration Cytology of Hepatoblastoma: Recognition of Subtypes on Cytomorphology. 2005; 49:355-364

Iyer VK, Verma K. Convoluted and Cleaved Nuclei in Rhabdomyosarcoma: An Unusual Morphologic Feature Causing Diagnostic Difficulty. 2005;49: 465-467 (Letter)

\section{J}

Jaffer S, Emanuel P, Burstein D, Goldfarb A. Cytologic Findings of Spindle Cell Ductal Carcinoma in Situ of the Breast: A Case Report. 2005;49:323-326 Jafri JA (see Madhavan et al). 2005;49:431-434

Jain A, Verma RK, Tiwari V, Goel MM. Dot-ELISA vs. PCR of Fine Needle Aspirates of Tuberculous Lymphadenitis: A Prospective Study in India. 2005;49:17-21

Jain P (see Sharma et al). 2005;49:335-338

Jain S (see Sharma et al). 2005;49:335-338

Jain S, Nigam S, Kumar N, Reddy BSN. Cutaneous Relapse in Hodgkin's Disease: A Case Report. 2005;49:191-194

Jalali M (see Gong et al). 2005;49:327-330

Jan C-I (see Nieh et al). 2005;49:31-37

Jannesar R (see Geramizadeh et al). 2005;49:666-668

Jang KS (see Oh et al). 2005;49:343-344 (Letter)

Jatiya L (see Kumar et al). 2005;49:111-112 (Letter)

Jayalakshmi P (see Jayaram et al). 2005;49:656-660

Jayaram G, Elsayed EM. Cytologic Evaluation of Prognostic Markers in Breast Carcinoma. 2005;49: 605-610

Jayaram G, Elsayed EM. Carcinosarcoma of the Palate: Report of a Case with a Diagnosis of Sarcomatoid Metastasis by Fine Needle Aspiration Cytology. 2005;49:520-524

Jayaram G, Jayalakshmi P, Yip CH. Leiomyosarcoma of the Breast: Report of a Case with Fine Needle Aspiration Cytologic, Histologic and Immunohistochemical Features. 2005;49:656-660

Jayaram G, Kaliaperumal S, Kumar G. Bilateral Carotid Body Tumor Diagnosed on Cytology. 2005;49:690-692 (Letter)

Jimbo H (see Kawana et al). 2005;49:507-512 
Jiménez-Heffernan JA (see Viguer et al). 2005;49:139_ 143

Jodra-Fernández O (see Martínez-Girón et al). 2005; 49:578-580 (Letter)

Jogai S, Al-Jassar A, Temmim L, Dey P, Adesina AO, Amanguno HG. Fine Needle Aspiration Cytology of the Thyroid: A Cytohistologic Study with Evaluation of Discordant Cases. 2005;49:483-488

Johnson DR (see Clary et al). 2005;49:378-382

Johnston DA (see Liu et al). 2005;49:157-162

Jordaan HF (see Peart et al). 2005;49:230-231 (Letter)

Joshi A (see Sah et al). 2005;49:349-350 (Letter)

Jungare A, Gadkari R, Lele VR, Bobhate SK. Utility of Papanicolaou Stain-Induced Fluorescence in the Cytodiagnosis of Extrapulmonary Tuberculosis. 2005;49:600-604

\section{$\mathbf{K}$}

Kaliaperumal S (see Jayaram et al). 2005;49:690-692 (Letter)

Kamath V (see Rao et al). 2005;49:249-256

Kamma H (see Fujiwara et al). 2005;49:575-578 (Letter)

Kapila K (see Iyer et al). 2005;49:355-364

Kaplanis K (see Malle et al). 2005;49:11-16

Kapur S (see Khalbuss and Kapur). 2005;49:226-228 (Letter)

Karimi M (see Geramizadeh et al). 2005;49:110-111 (Letter)

Katz R (see Liu et al). 2005;49:157-162

Kawada T (see Yamaguchi et al). 2005;49:207-212

Kawana K, Yamada M, Jimbo H, Shirai T, Takahashi M, Sano Y, Shiromizu K. Diagnostic Usefulness of Endometrial Aspiration Cytology for Endometrial Cancer Cases with Normal Curettage Findings. 2005;49:507-512

Kaya D (see Demirezen et al). 2005;49:257-261

Kearney KA (see Sawaya et al). 2005;49:391-397

Khajeh F (see Kumar et al). 2005;49:177-180

Khalbuss WE, Kapur S. Cytomorphology of Laser/ Thermal Artifact in Ductal Cells in a Post-Laser Surgery Bile Duct Brushing Specimen. 2005;49: 226-228 (Letter)

Khan N (see Afroz et al). 2005;49:697-698 (Letter)

Kheirandish P (see Geramizadeh et al). 2005;49:666668

Khirwadkar N, Clark AH. Fine Needle Aspiration Cytology of Tubular Carcinoma of the Breast. 2005;49:344-345 (Letter)

Khullar U (see Handa et al). 2005;49:421-423

Kilpatrick SE (see Rinas et al). 2005;49:554-559

Kim JM (see Kim et al). 2005;49:644-649

Kim L, Park IS, Han JY, Kim JM, Chu YC. Aspira- tion Cytology of Fibrosarcomatous Variant of Dermatofibrosarcoma Protuberans with Osteoclastlike Giant Cells in the Chest Wall: A Case Report. 2005;49:644-649

Kim SH, Kim TS. Squash Smear Findings of Eosinophilic Granular Bodies in Pilocytic Astrocytoma. 2005;49:112-114 (Letter)

Kim TS (see Kim and Kim). 2005;49:112-114 (Letter)

Kinney W (see Sawaya et al). 2005;49:391-397

Kiyozuka Y, Koyama H, Nakata M, Matsuyama T, Nikaido Y, Shimano N, Tsubura A. Diagnostic Cytopathology in Type II Angiosarcoma of the Breast: A Case Report. 2005;49:560-566

Kojima M (see Ohta et al). 2005;49:181-186

Kok LP (see Boon et al). 2005;49:513-519

Kondi-Pafiti A (see Chatzistamou et al). 2005;49:108109 (Letter)

Koss LG. On Decoy Cells. 2005;49:233-234

Koutselini H (see Chatzistamou et al). 2005;49:108109 (Letter)

Koyama H (see Kiyozuka et al). 2005;49:560-566

Köybaşığlu F, Şimşek GG, Önal BÜ, Han Ü, Adabağ A. Oropharyngeal Chordoma Diagnosed by Fine Needle Aspiration: A Case Report. 2005;49: 173-176

Krassilnik N (see Wiriosuparto et al). 2005;49:309_ 313

Kren L, Rotterova P, Hermanova M, Krenova Z, Sterba J, Dvorak K, Goncharuk V, Wilner GD, McKenna BJ. Chylothorax as a Possible Diagnostic Pitfall: A Report of 2 Cases with Cytologic Findings. 2005;49:441-444

Krenova Z (see Kren et al). 2005;49:441-444

Kumar A (see Loya et al). 2005;49:97-100

Kumar G (see Jayaram et al). 2005;49:690-692 (Letter)

Kumar N (see Jain et al). 2005;49:191-194

Kumar N (see Sharma et al). 2005;49:335-338

Kumar P (see Garbyal et al). 2005;49:58-60

Kumar PV (see Daneshbod et al). 2005;49:331-334

Kumar PV (see Daneshbod et al). 2005;49:7-10

Kumar PV (see Shekarkhar et al). 2005;49:533-536

Kumar PV, Monabati A, Tabei SZ, Ramzy M, Husseini SV, Khajeh F. Metastatic Follicular Thyroid Carcinoma Diagnosed by Fine Needle Aspiration Cytology: A Report of 3 Cases. 2005;49:177-180

Kumar PV, Vasei M, Daneshbod Y, Zakerinia M, Ramzi M, Noorani H, Bagheri H, Talei AR, Soleimanpour H. Breast Myeloma: A Report of 3 Cases with Fine Needle Aspiration Cytologic Findings. 2005;49:445-448

Kumar S, Ferns S, Sujatha S, Jatiya L. Acid-Fast Staining Patterns and Their Correlation with HIV Positivity. 2005;49:111-112 (Letter)

Kunimura T (see Nakashima et al). 2005;49:87-91 
Kushima M (see Nakashima et al). 2005;49:87-91

Kwon MS. Aspiration Cytology of Mediastinal Seminoma: Report of a Case with Emphasis on the Diagnostic Role of Aspiration Cytology, Cell Block and Immunocytochemistry. 2005;49:669-672

Kwon MS. Aspiration Cytology of Pulmonary Small Cell Variant of Poorly Differentiated Synovial Sarcoma Metastatic from the Tongue: A Case Report. 2005;49:92-96

\section{$\mathbf{L}$}

Laforga JB. Malignant Epithelioid Gastrointestinal Stromal Tumors: Report of a Case with Cytologic and Immunohistochemical Studies. 2005;49:435440

Leclerc C (see Duboucher et al). 2005;49:273-277

Lee W-H (see Nieh et al). 2005;49:31-37

Lele VR (see Jungare et al). 2005;49:600-604

Lepore M (see Fulciniti et al). 2005;49:495-499

Lewin DN (see Hoda et al). 2005;49:199-203

Lima TP (see Longatto-Filho et al). 2005;49:500-506

Liu J, Katz R, Shin HJC, Johnston DA, Zhang H-Z, Caraway NP. Use of Mailed Urine Specimens in Diagnosing Urothelial Carcinoma by Cytology and DNA Image Analysis. 2005;49:157-162

Liu S (see Guo et al). 2005;49:132-138

Liu Y (see Clary et al). 2005;49:378-382

Longatto Filho A (see Mattosinho de Castro Ferraz et al). 2005;49:639-643

Longatto-Filho A, Maeda MYS, Eržen M, Branca M, Roteli-Martins C, Naud P, Derchain SFM, Hammes L, Matos J, Gontijo R, Sarian LO, Lima TP, Tatti S, Syrjänen S, Syrjänen K. Conventional Pap Smear and Liquid-Based Cytology as Screening Tools in Low-Resource Settings in Latin America: Experience of the Latin American Screening Study. 2005;49:500-506

López JI (see Andres et al). 2005;49:462-463 (Letter)

López-Ferrer P (see Viguer et al). 2005;49:139-143

Loya AC, Prayaga AK, Sundaram C, Shantharam V, Kumar A. Cytologic Diagnosis of Pancreatic Tuberculosis in Immunocompetent and Immunocompromised Patients: A Report of 2 Cases. 2005; 49:97-100

\section{M}

MacAulay C (see Cantor et al). 2005;49:405-415

Madhavan M, George PJ, Jafri JA, Idris Z. Intraventricular Squamous Papillary Craniopharyngioma: Report of a Case with Intraoperative Imprint Cytology. 2005;49:431-434
Maeda MYS (see Longatto-Filho et al). 2005;49:500 506

Malhotra P, Tandon R, Singh N, Arora VK, Bhatia A. Cytoplasmic Processes: A Distinct Cytomorphologic Feature of Langerhans Cell Histiocytosis. 2005;49:580-582 (Letter)

Malle D, Valeri R-M, Photiou C, Kaplanis K, Andreadis C, Tsavdaridis D, Destouni C. Significance of Immunocytochemical Expression of E-Cadherin, N-Cadherin and CD44 in Serous Effusions Using Liquid-Based Cytology. 2005;49: $11-16$

Maly A (see Meir et al). 2005;49:195-198

Maly B (see Meir et al). 2005;49:195-198

Mansouri D (see Mrad et al). 2005;49:427-430

Martin L (see Guo et al). 2005;49:132-138

Martinez EZ (see Amaral et al). 2005;49:244-248

Martínez-Girón R, Jodra-Fernández O, TormoMolina R, Esteban J-G, Ribas-Barceló A. Uncommon Structures Simulating Helminth Eggs in Sputum. 2005;49:578-580 (Letter)

Martínez-Parra D (see Robles-Frías et al). 2005;49: $149-153$

Maruyama R (see Takahashi et al). 2005;49:319-322

Mathur S, Sood R, Aron M, Iyer VK, Verma K. Cytologic Diagnosis of Pulmonary Nocardiosis: A Report of 3 Cases. 2005;49:567-570

Mathur S, Verma K. Peripheral T-Cell Lymphoma Not Otherwise Specified vs. Hodgkin's Lymphoma on Fine Needle Aspiration Cytology. 2005;49:373377

Matos J (see Longatto-Filho et al). 2005;49:500-506

Matsutani M (see Inagawa et al). 2005;49:452-456

Matsu-ura Y (see Takahashi et al). 2005;49:319-322

Matsuyama T (see Kiyozuka et al). 2005;49:560-566

Mattosinho de Castro Ferraz MdG, Dall' Agnol M, di Loreto C, Pirani WM, Utagawa ML, Pereira SMM, Sakai YI, Feres CL, Shih LWS, Yamamoto LS, Rodrigues ROL, Shirata NK, Longatto Filho A. 100\% Rapid Rescreening for Quality Assurance in a Quality Control Program in a Public Health Cytologic Laboratory. 2005;49:639-643

McGregor DK (see Sun et al). 2005;49:525-529

McKenna BJ (see Kren et al). 2005;49:441-444

Mehta A, Nema SK, Nakra R, Patkar N. New Cytologic Features of Solid and Papillary Epithelial Neoplasms of the Pancreas. 2005;49:696-697 (Letter)

Meir K, Maly B, Shoshan Y, Maly A, Soffer D. Cerebral Amyloidoma Diagnosed Intraoperatively with Squash Preparations: A Case Report. 2005;49:195198

Menéndez CL (see Velasco et al). 2005;49:585-586 (Letter)

Menendez CLM (see Pinto Blázquez et al). 2005;49: 
653-655

Miller KB (see Pantanowitz et al). 2005;49:583-584

(Letter)

Miller MG (see Sawaya et al). 2005;49:391-397

Minamiguchi S (see Hoda et al). 2005;49:199-203

Mitsuhashi T (see Inagawa et al). 2005;49:452-456

Mitsuya T (see Ikeda et al). 2005;49:314-318

Miura H (see Takahashi et al). 2005;49:319-322

Mohan H (see Handa et al). 2005;49:421-423

Monabati A (see Daneshbod et al). 2005;49:331-334

Monabati A (see Daneshbod et al). 2005;49:7-10

Monabati A (see Kumar et al). 2005;49:177-180

Monga G (see Boldorini et al). 2005;49:235-243

Monga G (see Bozzola et al). 2005;49:213-218

Montemor EB (see Amaral et al). 2005;49:244-248

Morales C (see Ravinsky and Morales). 2005;49:51-57

Morohoshi T (see Nakashima et al). 2005;49:87-91

Morosini P (see Branca et al). 2005;49:398-404

Mrad K, Mansouri D, Driss M, Sassi S, Abbes I, Ayed FB, Ben Romdhane K. Esthesioneuroblastoma Metastatic to the Breast in a Young Woman: A Case Report. 2005;49:427-430

Munshi M (see Deshpande and Munshi). 2005;49:8186

Musso C, Silva-Santos MC, Pereira FEL. Cotton Block Method: One-Step Method of Cell Block Preparation After Fine Needle Aspiration. 2005;49: 22-26

\section{$\mathbf{N}$}

Nakamura S (see Uzaslan et al). 2005;49:154-156 [erratum 2005;49(5):127A]

Nakashima M, Inagaki T, Kunimura T, Kushima M, Adachi M, Morohoshi T. Cytopathologic and Histologic Features of Biphasic Pulmonary Blastoma: A Case Report. 2005;49:87-91

Nakata M (see Kiyozuka et al). 2005;49:560-566

Nakayama K (see Yamaguchi et al). 2005;49:207-212

Nakra R (see Mehta et al). 2005;49:696-697 (Letter)

Naldi N (see Nizzoli et al). 2005;49:27-30

Naud P (see Longatto-Filho et al). 2005;49:500-506

Nazeer S (see Akinremi et al). 2005;49:169-172

Nema SK (see Mehta et al). 2005;49:696-697 (Letter)

$\mathrm{Ng}$ B (see Baehner et al). 2005;49:543-548

$\mathrm{Ng}$ SB (see Chuah et al). 2005;49:75-80

Nguyen G-K (see Batoroev and Nguyen). 2005;49: 464-465 (Letter)

Nieh S, Chen S-F, Fu E, Jan C-I, Lee W-H. Detection of the Human Telomerase RNA Component by in Situ Hybridization in Cells from Body Fluids. 2005;49:31-37

Nigam S (see Jain et al). 2005;49:191-194

Nikaido Y (see Kiyozuka et al). 2005;49:560-566
Nishikawa R (see Inagawa et al). 2005;49:452-456

Nizzoli R, Guazzi A, Naldi N, Franciosi V, Bozzetti C. HER-2/neu Evaluation by Fluorescence in Situ Hybridization on Destained Cytologic Smears from Primary and Metastatic Breast Cancer. 2005;49:27-30

Noël C (see Duboucher et al). 2005;49:273-277

Noorani H (see Kumar et al). 2005;49:445-448

\section{0}

O'Connell F, Cibas ES. Cytologic Features of Ciliated Adenocarcinoma of the Cervix: A Case Report. 2005;49:187-190

Oh Y-H, Jang KS, Song YS, Paik SS, Park YW, Chon S-H. Secretory Carcinoma of the Breast Diagnosed by Fine Needle Aspiration. 2005;49:343-344 (Letter)

Ohta Y, Shiokawa A, Suzuki T, Kojima M. Cytology, Immunohistochemistry and 3-Dimensional Reconstruction of Adenoma Malignum: A Case Report. 2005;49:181-186

Oliveira AM, French CA. Applications of Fluorescence in Situ Hybridization in Cytopathology: A Review. 2005;49:587-594

Önal BÜ (see Köybaşıŏlu et al). 2005;49:173-176

Ordóñez NG (see Gong et al). 2005;49:537-542

Ordonez NG (see Sun et al). 2005;49:525-529

Otal-Salaverri C (see Robles-Frías et al). 2005;49:149_ 153

Ouwerkerk-Noordam E (see Boon et al). 2005;49: 513-519

Oyama T (see Hara et al). 2005;49:43-50

Oyama T (see Hara et al). 2005;49:611-620

Ozdemir G (see Bakaris et al). 2005;49:1-6

Ozdemir M (see Bakaris et al). 2005;49:1-6

Paik SS (see Oh et al). 2005;49:343-344 (Letter)

Paiva RL (see Bohrer et al). 2005;49:265-272

Palombini L (see Fulciniti et al). 2005;49:495-499

Pandidou A (see Valeri et al). 2005;49:61-64

Panikar N, Sikka M, Singh N. Concurrent Chronic Myelogenous Leukemia and Tuberculous Lymphadenitis: A Case Report. 2005;49:650-652

Pantanowitz L, Miller KB, Pihan G. Cytology of "Pseudoseptic" Leukemic Arthritis. 2005;49:583584 (Letter)

Park IS (see Kim et al). 2005;49:644-649

Park YW (see Oh et al). 2005;49:343-344 (Letter)

Patkar N (see Mehta et al). 2005;49:696-697 (Letter)

Paulino AFG (see Afify et al). 2005;49:621-626 
Peart LK, Schneider JW, Jordaan HF, Wright CA. Fine Needle Aspiration Biopsy of Postvaccination Disseminated Mycobacterium bovis Infection Presenting as a Solitary Cutaneous Papule. 2005;49: 230-231 (Letter)

Pereira FEL (see Musso et al). 2005;49:22-26

Pereira SMM (see Mattosinho de Castro Ferraz et al). 2005;49:639-643

Pettenati MJ (see Taylor et al). 2005;49:673-676

Pezerović-Panijan R (see Banek et al). 2005;49:627633

Pham S (see Duboucher et al). 2005;49:273-277

Photiou C (see Malle et al). 2005;49:11-16

Pihan G (see Pantanowitz et al). 2005;49:583-584 (Letter)

Pinheiro DL (see Collaço et al). 2005;49:694-696 (Letter)

Pinto Blázquez J, Velasco Alonso J, Menendez CLM, Alonso de la Campa J, Astudillo Gonzalez A. Fine Needle Aspiration Cytology of Focal Myositis: A Case Report. 2005;49:653-655

Pinto J (see Velasco et al). 2005;49:585-586 (Letter)

Pirani WM (see Mattosinho de Castro Ferraz et al). 2005;49:639-643

Poh WT (see Chuah et al). 2005;49:75-80

Politi E (see Chatzistamou et al). 2005;49:108-109 (Letter)

Ponder TB (see Ezenekwe et al). 2005;49:549-553

Prasad R (see Sah et al). 2005;49:286-290

Prayaga AK (see Loya et al). 2005;49:97-100

\section{Q}

Qureshi MN, Bolick D, Ringer PJ, Spangler FL, Zimmerman G. HPV Testing in Liquid Cytology Specimens: Comparison of Analytic Sensitivity and Specificity for in Situ Hybridization and Chemiluminescent Nucleic Acid Testing. 2005;49:120-126

Qureshi MN. Detecting High-Risk Human Papillomavirus in Liquid-Based Cytology Specimens. 2005;49:582 (Letter)

\section{$\mathbf{R}$}

Raab SS (see Clary et al). 2005;49:378-382

Rados PV (see Bohrer et al). 2005;49:265-272

Raj GA (see Sah et al). 2005;49:286-290

Ramponi A (see Bozzola et al). 2005;49:213-218

Ramrakhiani D (see Anuradha et al). 2005;49:661-665

Ramzi M (see Kumar et al). 2005;49:445-448

Ramzy M (see Kumar et al). 2005;49:177-180

Rao ACK (see Rao et al). 2005;49:249-256

Rao J (see Wiriosuparto et al). 2005;49:309-313
Rao L, Kamath V, Boon ME, Valiathan M, Rao ACK. Pap Smear Quality Parameters of 3 Sampling Devices Used by Auxiliary Nurse Midwives on Symptomatic Rural Indian Women. 2005;49:249256

Rastegar M (see Daneshbod et al). 2005;49:331-334

Ravinsky E, Morales C. Diagnosis of Lymphoma by Image-Guided Needle Biopsies: Fine Needle Aspiration Biopsy, Core Biopsy or Both? 2005;49:51-57

Reddy BSN (see Jain et al). 2005;49:191-194

Ren R (see Gong et al). 2005;49:537-542

Ribas-Barceló A (see Martínez-Girón et al). 2005;49: 578-580 (Letter)

Rinas AC, Ward WG, Kilpatrick SE. Potential Sampling Error in Fine Needle Aspiration Biopsy of Dedifferentiated Chondrosarcoma: A Report of 4 Cases. 2005;49:554-559

Ringer PJ (see Qureshi et al). 2005;49:120-126

Rio ED (see Suárez-Peñaranda et al). 2005;49:530532

Robles-Frías A, González-Cámpora R, MartínezParra D, Robles-Frías MJ, Vázquez-Cerezuela T, Otal-Salaverri C, Fernández-Palacín A. Robinson Cytologic Grading of Invasive Ductal Breast Carcinoma: Correlation with Histologic Grading and Regional Lymph Node Metastasis. 2005;49:149_ 153

Robles-Frías MJ (see Robles-Frías et al). 2005;49:149_ 153

Rodrigues ROL (see Mattosinho de Castro Ferraz et al). 2005;49:639-643

Roteli-Martins C (see Longatto-Filho et al). 2005;49: 500-506

Rotterova P (see Kren et al). 2005;49:441-444

Roy S (see Garbyal et al). 2005;49:58-60

\section{S}

Sabo E (see Szporn et al). 2005;49:387-390

Sah SP (see Bhadani et al). 2005;49:114-116 (Letter)

Sah SP (see Bhadani et al). 2005;49:228-230 (Letter)

Sah SP (see Bhadani et al). 2005;49:347-348 (Letter)

Sah SP, Joshi A, Hansdak SG. Fine Needle Aspiration of Precursor B Lymphoblastic Leukemia in an Adult Presenting as Disseminated Skin Nodules. 2005;49:349-350 (Letter)

Sah SP, Prasad R, Raj GA. Fine Needle Aspiration of Lymphadenopathy in Visceral Leishmaniasis. 2005;49:286-290

Sakai YI (see Mattosinho de Castro Ferraz et al). 2005;49:639-643

Sano Y (see Kawana et al). 2005;49:507-512

Sarian LO (see Longatto-Filho et al). 2005;49:500506 
Sassi S (see Mrad et al). 2005;49:427-430

Satoh H (see Fujiwara et al). 2005;49:575-578 (Letter) Sawaya GF, Sung H-Y, Kinney W, Kearney KA, Miller MG, Hiatt RA. Cervical Cancer After Multiple Negative Cytologic Tests in Long-Term Members of a Prepaid Health Plan. 2005;49:391397

Schally AV (see Chatzistamou et al). 2005;49:108-109 (Letter)

Schneider JW (see Peart et al). 2005;49:230-231 (Letter)

Selvaggi SM. Cytologic Features of High Grade Squamous Intraepithelial Lesions Involving Endocervical Glands on Thin-Layer Cytology. 2005;49: 689-690 (Letter)

Severi P (see Branca et al). 2005;49:398-404

Shantharam V (see Loya et al). 2005;49:97-100

Sharma P, Kumar N, Jain P, Gur R, Jain S. Chronic Wuchereriasis Presenting as a Vaginoperineal Fistula: Report of a Case with Aspiration Cytologic Diagnosis. 2005;49:335-338

Sharma S (see Anshu et al). 2005;49:204-206

Sharma S (see Sodhani et al). 2005;49:634-638

Sharma U (see Bhadani et al). 2005;49:228-230 (Letter)

Shekarkhar MJ, Tabei SZ, Kumar PV, Hashemi SB. Cytologic Findings in Calcifying Epithelial Odontogenic Tumor: A Case Report. 2005;49:533-536

Shet T (see Bavi et al). 2005;49:424-426

Shih LWS (see Mattosinho de Castro Ferraz et al). 2005;49:639-643

Shih S-R, Shun C-T, Su D-H, Hsiao Y-L, Chang T-C. Follicular Variant of Papillary Thyroid Carcinoma: Diagnostic Limitations of Fine Needle Aspiration Cytology. 2005;49:383-386

Shih S-R, Su D-H, Hsiao Y-L, Chang T-C. Reactive Plasmacytosis of the Thyroid Gland in a Patient with Disseminated Tuberculosis. 2005;49:584-585 (Letter)

Shimano N (see Kiyozuka et al). 2005;49:560-566

Shimizu M (see Inagawa et al). 2005;49:452-456

Shin HJC (see Liu et al). 2005;49:157-162

Shiokawa A (see Ohta et al). 2005;49:181-186

Shirai T (see Kawana et al). 2005;49:507-512

Shirata NK (see Mattosinho de Castro Ferraz et al). 2005;49:639-643

Shiromizu K (see Kawana et al). 2005;49:507-512

Shivkumar VB (see Anshu et al). 2005;49:204-206

Shoshan Y (see Meir et al). 2005;49:195-198

Shun C-T (see Shih et al). 2005;49:383-386

Sikka M (see Panikar et al). 2005;49:650-652

Silva-Santos MC (see Musso et al). 2005;49:22-26

Simons DP (see Zardawi et al). 2005;49:224-226 (Letter)

Şimşek GG (see Köybaşığlu et al). 2005;49:173-176
Singh A, Singh OP, Goel MM. Frozen Shoulder as the Initial Presentation of Gallbladder Cancer Diagnosed by Fine Needle Aspiration Cytology. 2005;49:345-347 (Letter)

Singh MM (see Sodhani et al). 2005;49:634-638

Singh N (see Malhotra et al). 2005;49:580-582 (Letter)

Singh N (see Panikar et al). 2005;49:650-652

Singh OP (see Singh et al). 2005;49:345-347 (Letter)

Singh RK (see Bhadani et al). 2005;49:347-348 (Letter)

Sinha A (see Anuradha et al). 2005;49:661-665

Sköld CM (see Elmberger et al). 2005;49:223-224 (Letter)

Skordalaki A (see Valeri et al). 2005;49:61-64

Smith ME (see Faulkner-Jones et al). 2005;49:297-308

Sneige N (see Gong et al). 2005;49:537-542

Sodhani P, Garg S, Bhalla P, Singh MM, Sharma S, Gupta S. Prevalence of Bacterial Vaginosis in a Community Setting and Role of the Pap Smear in Its Detection. 2005;49:634-638

Soffer D (see Meir et al). 2005;49:195-198

Soleimanpour H (see Kumar et al). 2005;49:445-448

Song YS (see Oh et al). 2005;49:343-344 (Letter)

Sood R (see Mathur et al). 2005;49:567-570

Soundararajan S (see Bewtra et al). 2005;49:127-131

Spangler FL (see Qureshi et al). 2005;49:120-126

Staerkel G (see Gong et al). 2005;49:327-330

Sterba J (see Kren et al). 2005;49:441-444

Stoler MH. HPV Testing in Cervical Cytology Practice: It's All About Choice. 2005;49:117-119

Su D-H (see Shih et al). 2005;49:383-386

Su D-H (see Shih et al). 2005;49:584-585 (Letter)

Suárez-Peñaranda JM, Antúnez JR, Rio ED, Vázquez VH, Domínguez AN. Vaginal Involvement in a Woman with Darier's Disease: A Case Report. 2005;49:530-532

Suda K (see Hara et al). 2005;49:43-50

Suda K (see Hara et al). 2005;49:611-620

Sudilovsky D (see Baehner et al). 2005;49:543-548

Sujatha S (see Kumar et al). 2005;49:111-112 (Letter)

Sun W, McGregor DK, Ordonez NG, Ayala AG, Caraway NP. Fine Needle Aspiration Cytology of a Low Grade Myxoid Renal Epithelial Neoplasm:

A Case Report. 2005;49:525-529

Sun X (see Gong et al). 2005;49:537-542

Sundaram C (see Loya et al). 2005;49:97-100

Sung H-Y (see Sawaya et al). 2005;49:391-397

Suurmeijer AJH (see Boon et al). 2005;49:513-519

Suzuki T (see Ikeda et al). 2005;49:314-318

Suzuki T (see Ohta et al). 2005;49:181-186

Syrjänen K (see Branca et al). 2005;49:398-404

Syrjänen K (see Longatto-Filho et al). 2005;49:500 506

Syrjänen S (see Longatto-Filho et al). 2005;49:500- 
506

Szporn A, Chen X, Wu M, Burstein DE. Collagen Balls in Cervical-Vaginal Smears. 2005;49:262-264 Szporn AH, Chen X, Wu M, Sabo E, Burstein DE. Increase in the Incidence of Peritoneal Collagen Balls Over a 10-Year Period. 2005;49:387-390

\section{$\mathbf{T}$}

Tabei SZ (see Kumar et al). 2005;49:177-180

Tabei SZ (see Shekarkhar et al). 2005;49:533-536

Tadros TS (see Bakotic et al). 2005;49:163-168

Taghipoor M (see Daneshbod et al). 2005;49:7-10

Takahashi M (see Kawana et al). 2005;49:507-512

Takahashi T, Miura H, Matsu-ura Y, Iwana S, Maruyama R, Harada T. Urine Cytology of Localized Primary Amyloidosis of the Ureter: A Case Report. 2005;49:319-322

Talei AR (see Kumar et al). 2005;49:445-448

Tan PH (see Chuah et al). 2005;49:75-80

Tandon R (see Malhotra et al). 2005;49:580-582 (Letter)

Tate G (see Ikeda et al). 2005;49:314-318

Tatti S (see Longatto-Filho et al). 2005;49:500-506

Taylor CA, Barnhart A, Pettenati MJ, Geisinger KR. Primary Pleuropulmonary Synovial Sarcoma Diagnosed by Fine Needle Aspiration with Cytogenetic Confirmation: A Case Report. 2005;49:673-676

Temmim L (see Jogai et al). 2005;49:483-488

Tewari A (see Bhadani et al). 2005;49:114-116 (Letter)

Tiwari V (see Jain et al). 2005;49:17-21

Tormo-Molina R (see Martínez-Girón et al). 2005; 49:578-580 (Letter)

Tötsch M (see Akinremi et al). 2005;49:169-172

Tsavdaridis D (see Malle et al). 2005;49:11-16

Tsubura A (see Kiyozuka et al). 2005;49:560-566

\section{$\mathbf{U}$}

Üçok C (see Üçok et al). 2005;49:38-42

Üçok Ö, Doğan N, Üçok C, Günhan Ö. Role of Fine Needle Aspiration Cytology in the Preoperative Presumptive Diagnosis of Ameloblastoma. 2005; 49:38-42

Uguz A, Ersoz C, Bolat F, Gokdemir A, Vardar MA. Fine Needle Aspiration Cytology of Ovarian Lesions. 2005;49:144-148

Utagawa ML (see Mattosinho de Castro Ferraz et al). 2005;49:639-643

Uzaslan E, Ebsen M, Freudenberg N, Nakamura S, Costabel U, Guzman J. Reactive Alveolar Epithelium in Chondroid Hamartoma of the Lung. 2005; 49:154-156 [erratum 2005;49(5):127A]
Vahtsevanos C (see Valeri et al). 2005;49:61-64

Valeri R-M (see Malle et al). 2005;49:11-16

Valeri R-M, Hadjileontis C, Skordalaki A, Pandidou A, Vahtsevanos C, Destouni H. Salivary Duct Carcinoma of the Parotid Gland: Report of a Rare Case with a Comparative Study of Aspiration Cytology and Histomorphology. 2005;49:61-64

Valiathan M (see Rao et al). 2005;49:249-256

Vardar MA (see Uguz et al). 2005;49:144-148

Vasei M (see Kumar et al). 2005;49:445-448

Vázquez VH (see Suárez-Peñaranda et al). 2005;49: $530-532$

Vázquez-Cerezuela T (see Robles-Frías et al). 2005; 49:149-153

Veggiani C (see Boldorini et al). 2005;49:235-243

Velasco Alonso J (see Pinto Blázquez et al). 2005;49: 653-655

Velasco J, Menéndez CL, de la Campa JA, Pinto J, Crespo M. Cyanoacrylate Hepatic Pseudotumor: Role of Fine Needle Aspiration. 2005;49:585-586 (Letter)

Verma K (see Iyer and Verma). 2005;49:465-467 (Letter)

Verma K (see Iyer et al). 2005;49:355-364

Verma K (see Mathur and Verma). 2005;49:373-377

Verma K (see Mathur et al). 2005;49:567-570

Verma RK (see Jain et al). 2005;49:17-21

Vetrani A (see Fulciniti et al). 2005;49:495-499

Vicandi B (see Viguer et al). 2005;49:139-143

Viguer JM, Jiménez-Heffernan JA, López-Ferrer P, González-Peramato P, Vicandi B. Fine Needle Aspiration of Toxoplasmic (Piringer-Kuchinka) Lymphadenitis: A Cytohistologic Correlation Study. 2005;49:139-143

Viscogliosi E (see Duboucher et al). 2005;49:273-277

\section{W}

Ward WG (see Rinas et al). 2005;49:554-559

Wasserman P (see Edwards and Wasserman). 2005; 49:489-494

Weselow G (see Hoda et al). 2005;49:199-203

Westin MCdA (see Amaral et al). 2005;49:244-248

Wilander E (see de Lang and Wilander). 2005;49:595599

Wilbur DC. The Annual Pap Test: Baseline Data to Allow Informed Decisions About Future Screening Programs. 2005;49:351-353

Wildi SM (see Hoda et al). 2005;49:199-203

Wilkinson EJ (see Brown and Wilkinson). 2005;49: 71-74

Willis D (see Bakotic et al). 2005;49:163-168

Wilner GD (see Kren et al). 2005;49:441-444 
Wiriosuparto S, Krassilnik N, Bhuta S, Rao J, Hirschowitz S. Solitary Fibrous Tumor: Report of a Case with an Unusual Presentation as a Spindle Cell Parotid Neoplasm. 2005;49:309-313

Wood MD, Huang Y, Bibbo M. Improving Recognition of Thyroid Carcinoma in Rapid-Consultation Specimens. 2005;49:291-296

Wright CA (see Peart et al). 2005;49:230-231 (Letter)

Wu M, Burstein DE. Emperipolesis in Pleural Mesothelial Cells in a Patient with Chronic Lymphocytic Leukemia. 2005;49:692-694 (Letter)

Wu M (see Szporn et al). 2005;49:262-264

Wu M (see Szporn et al). 2005;49:387-390

Wu S-L (see Chang et al). 2005;49:477-482

\section{$\mathbf{x}$}

Xie Q (see Bewtra et al). 2005;49:127-131

\section{$\mathbf{Y}$}

Yamada M (see Kawana et al). 2005;49:507-512
Yamaguchi T, Imamura Y, Nakayama K, Kawada T, Yamamoto T, Fukuda M. Paranuclear Blue Inclusions of Small Cell Carcinoma of the Stomach: Report of a Case with Cytologic Presentation in Peritoneal Washings. 2005;49:207-212

Yamamoto LS (see Mattosinho de Castro Ferraz et al). 2005;49:639-643

Yamamoto T (see Yamaguchi et al). 2005;49:207-212

Yip CH (see Jayaram et al). 2005;49:656-660

\section{Z}

Zakerinia M (see Kumar et al). 2005;49:445-448

Zardawi IM, Barker BJ, Simons DP. Hodgkin's Disease Masquerading as Granulomatous Lymphadenitis on Fine Needle Aspiration Cytology. 2005; 49:224-226 (Letter)

Zeferino LC (see Amaral et al). 2005;49:244-248

Zhang H-Z (see Liu et al). 2005;49:157-162

Zhao M (see Gu et al). 2005;49:471-476

Zhou H (see Afify et al). 2005;49:621-626

Zimmerman G (see Qureshi et al). 2005;49:120-126 